\title{
EXISTENCE AND MULTIPLICITY OF NORMALIZED SOLUTIONS FOR THE NONLINEAR CHERN-SIMONS-SCHRÖDINGER EQUATIONS
}

\author{
Haibo Chen and Weihong Xie \\ Central South University, School of Mathematics and Statistics \\ Changsha, Hunan 410083, P. R. China; math_chb@163.com \\ Central South University, School of Mathematics and Statistics \\ Changsha, Hunan 410083, P. R. China; xieweihong0218@163.com
}

\begin{abstract}
In this paper, we prove the existence and multiplicity results of solutions with prescribed $L^{2}$-norm for a class of nonlinear Chern-Simons-Schrödinger equations in $\mathbf{R}^{2}$

$$
-\Delta u-\lambda u+\kappa\left(\frac{h^{2}(|x|)}{|x|^{2}}+\int_{|x|}^{\infty} \frac{h(s)}{s} u^{2}(s) d s\right) u=f(u),
$$

where $\lambda \in \mathbf{R}, \kappa>0, f \in \mathcal{C}(\mathbf{R}, \mathbf{R})$ and

$$
h(s)=\frac{1}{2} \int_{0}^{s} r u^{2}(r) d r .
$$

To obtain such solutions, we look into critical points of the energy functional

$$
E_{\kappa}(u)=\frac{1}{2} \int_{\mathbf{R}^{2}}|\nabla u|^{2}+\frac{\kappa}{2} \int_{\mathbf{R}^{2}} \frac{|u|^{2}}{|x|^{2}}\left(\int_{0}^{|x|} \frac{r}{2} u^{2}(r) d r\right)^{2}-\int_{\mathbf{R}^{2}} F(u)
$$

constrained on the $L^{2}$-spheres $S_{r}(c)=\left\{u \in H_{r}^{1}\left(\mathbf{R}^{2}\right):\|u\|_{2}^{2}=c\right\}$. Here, $c>0$ and $F(s):=\int_{0}^{s} f(t) d t$. Under some mild assumptions on $f$, we show that critical points of $E_{\kappa}$ unbounded from below on $S_{r}(c)$ exist for certain $c>0$. In addition, we establish the existence of infinitely many critical points $\left\{u_{n}^{\kappa}\right\}$ of $E_{\kappa}$ on $S_{r}(c)$ provided that $f$ is odd. Finally, we regard $\kappa$ as a parameter and and present a convergence property of $u_{n}^{\kappa}$ as $\kappa \searrow 0$. These results improve and generalize the existing ones in the literature.
\end{abstract}

\section{Introduction}

Jackiw and $\mathrm{Pi}$ in $[13,14]$ introduced a nonrelativistic model that the nonlinear Schrödinger dynamics is coupled with the Chern-Simons gauge terms as follows:

$$
\left\{\begin{array}{l}
i D_{0} \phi+\left(D_{1} D_{1}+D_{2} D_{2}\right) \phi=-|\phi|^{p-2} \phi, \\
\partial_{0} A_{1}-\partial_{1} A_{0}=-\operatorname{Im}\left(\bar{\phi} D_{2} \phi\right) \\
\partial_{0} A_{2}-\partial_{2} A_{0}=\operatorname{Im}\left(\bar{\phi} D_{1} \phi\right) \\
\partial_{1} A_{2}-\partial_{2} A_{1}=-\frac{1}{2}|\phi|^{2}
\end{array}\right.
$$

where $i$ denotes the imaginary unit, $\partial_{0}=\frac{\partial}{\partial t}, \partial_{1}=\frac{\partial}{\partial x_{1}}, \partial_{2}=\frac{\partial}{\partial x_{2}}$ for $\left(t, x_{1}, x_{2}\right) \in \mathbf{R}^{1+2}$, $\phi: \mathbf{R}^{1+2} \rightarrow \mathbf{C}$ is a complex scalar filed, $A_{\mu}: \mathbf{R}^{1+2} \rightarrow \mathbf{R}$ is the gauge filed and $D_{\mu}=$ $\partial_{\mu}+i A_{\mu}$ is the covariant derivative for $\mu$ running over $0,1,2$. The Chern-Simons gauge theory describes the nonrelativistic thermodynamic behavior of large number of particles in an electromagnetic field. This feature of the model is important for

https://doi.org/10.5186/aasfm.2020.4518

2010 Mathematics Subject Classification: Primary 35J20, 35J60.

Key words: Chern-Simons-Schrödinger equations, normalized solutions, multiplicity results, asymptotic behavior. 
the study of the high temperature superconductor, Aharovnov-Bohm scattering and the fractional quantum Hall effect.

The system (1.1) is invariant under the following gauge transformation

$$
\phi \mapsto \phi e^{i \chi}, \quad A_{\mu} \mapsto A_{\mu}-\partial_{\mu} \chi,
$$

where $\chi: \mathbf{R}^{1+2} \rightarrow \mathbf{R}$ is an arbitrary $\mathcal{C}^{\infty}$ function. Recently, the existence of stationary states for system (1.1) has been extensively investigated, see for example [8, 9, 10, $11,12,17,15,22,23,28,29,32]$. In these references, the authors seek the solutions to (1.1) of the following form

$$
\begin{aligned}
\phi(t, x) & =u(|x|) e^{-i \lambda t}, & & A_{0}(t, x)=k(|x|), \\
A_{1}(t, x) & =\frac{x_{2}}{|x|^{2}} h(|x|), & & A_{2}(t, x)=-\frac{x_{1}}{|x|^{2}} h(|x|) .
\end{aligned}
$$

If we insert the ansatz (1.2) into the system (1.1), then (1.1) is reduced to the following nonlocal elliptic equation:

$$
-\Delta u-\lambda u+\left(\frac{h^{2}(|x|)}{|x|^{2}}+\int_{|x|}^{\infty} \frac{h(s)}{s} u^{2}(s) d s\right) u=|u|^{p-2} u, x \in \mathbf{R}^{2},
$$

where $h(s)=\frac{1}{2} \int_{0}^{s} r u^{2}(r) d r$. For more details about (1.1)-(1.3), we refer the readers to $[8,9,11,22,23,25]$.

In the present paper, motivated by the fact that physicists often seek "normalized" solutions, we search for solutions with prescribed $L^{2}$-norm of the problem (1.3) with a general nonlinearity:

$$
-\Delta u-\lambda u+\kappa\left(\frac{h^{2}(|x|)}{|x|^{2}}+\int_{|x|}^{\infty} \frac{h(s)}{s} u^{2}(s) d s\right) u=f(u), \quad x \in \mathbf{R}^{2},
$$

where $\lambda \in \mathbf{R}, \kappa>0$ and $f$ verifies the following assumptions:

$\left(f_{1}\right) f \in \mathcal{C}(\mathbf{R}, \mathbf{R})$ and $f(t)=o(|t|)$ as $t \rightarrow 0$;

$\left(f_{2}\right)$ there exists $p>4$ such that $f(t) t \leq p F(t)$ for all $t \in \mathbf{R}$, where $F(t):=$ $\int_{0}^{t} f(s) d s$

$\left(f_{3}\right) \lim _{|t| \rightarrow \infty} \frac{F(t)}{t^{4}}=\infty$

$\left(f_{4}\right)$ the function $\frac{f(t) t-2 F(t)}{|t|^{3} t}$ is strictly increasing on $(-\infty, 0) \cup(0, \infty)$;

$\left(f_{5}\right) f$ is odd.

Under the above conditions, it is well known (see $[3,16]$ ) that a solution of $(1.4)$ with $\|u\|_{2}^{2}=c$ can be obtained as a constrained critical point of the functional

$$
E_{\kappa}(u)=\frac{1}{2} \int_{\mathbf{R}^{2}}|\nabla u|^{2}+\frac{\kappa}{2} \int_{\mathbf{R}^{2}} \frac{|u|^{2}}{|x|^{2}}\left(\int_{0}^{|x|} \frac{r}{2} u^{2}(r) d r\right)^{2}-\int_{\mathbf{R}^{2}} F(u)
$$

on the constraint

$$
S_{r}(c)=\left\{u \in H_{r}^{1}\left(\mathbf{R}^{2}\right):\|u\|_{2}^{2}=c, c>0\right\} .
$$

The frequency $\lambda$, in this situation, can not be fixed any more and it appears as a Lagrange parameter with respect to the constraint $S_{r}(c)$.

More recently, normalized solutions for elliptic equations have received much attention. See e.g. [1, 2, 3, 4, 5, 18, 19, 20, 21, 34]. Let we state some known results. In [18], Jeanjean considered the following nonlinear Schrödinger equation:

$$
-\Delta u-\lambda u=f(u), \quad \lambda \in \mathbf{R}, \quad x \in \mathbf{R}^{N},
$$

where the following hypotheses on $f$ are introduced: 
$\left(H_{1}\right) f: \mathbf{R} \rightarrow \mathbf{R}$ is continuous and odd;

$\left(H_{2}\right)$ there exist $\alpha, \beta \in \mathbf{R}$ satisfying $2+\frac{4}{N}<\alpha \leq \beta<2^{*}$ such that

$$
0<\alpha F(s) \leq f(s) s \leq \beta F(s), \quad \forall s \in \mathbf{R} \backslash\{0\},
$$

where $2^{*}=2 N /(N-2)$ if $N \geq 3$ and $2^{*}=\infty$ if $N=2$.

The condition $F(s)>0$ in $\left(H_{2}\right)$ is not stated in [18] but used implicitly. Then it is proved that (1.6) admits a couple of solutions $\left(u_{c}, \lambda_{c}\right) \in H_{r}^{1}\left(\mathbf{R}^{N}\right) \times \mathbf{R}^{-}$with $\left\|u_{c}\right\|_{2}^{2}=c$ for $N \geq 2$. Moreover, the author also indicated the bifurcation result associated with (1.6), that is,

$$
\left\|\nabla u_{c}\right\|_{2} \rightarrow \infty, \lambda_{c} \rightarrow-\infty \text { as } c \rightarrow 0 ; \quad\left\|\nabla u_{c}\right\|_{2} \rightarrow 0, \lambda_{c} \rightarrow 0 \text { as } c \rightarrow \infty .
$$

Later, if $\left(H_{1}\right)$ and $\left(H_{2}\right)$ are satisfied, Bartsch and De Valeriola [1] obtained the existence of infinitely many normalized solutions for (1.6).

In [3], Bellazzini et al. dealt with the following Schrödinger-Poisson equation:

$$
-\Delta u-\lambda u+\left(|x|^{-1} * u^{2}\right) u-|u|^{q-2} u=0, \quad x \in \mathbf{R}^{3} .
$$

By using a mountain pass argument developed on

$$
S(c)=\left\{u \in H^{1}\left(\mathbf{R}^{3}\right):\|u\|_{2}^{2}=c\right\}, \quad c>0,
$$

they established the existence of $\left(u_{c}, \lambda_{c}\right) \in S(c) \times \mathbf{R}^{-}$a couple of solutions of (1.8) for $c>0$ sufficiently small and $q \in\left(\frac{10}{3}, 6\right)$. For the case $q \in\left(2, \frac{10}{3}\right]$, we refer the readers to $[4,5,19]$. Afterwards, based on [1, 3], Luo [20] has demonstrated that when $q \in\left(\frac{10}{\underline{3}}, 6\right)$, problem (1.8) admits an unbounded sequence of couples of solutions $\left(u_{n}, \lambda_{n}\right) \in \bar{S}_{r}(c) \times \mathbf{R}^{-}$for each $n \in \mathbf{N}^{+}$, where $\bar{S}_{r}(c)=\left\{u \in H_{r}^{1}\left(\mathbf{R}^{3}\right):\|u\|_{2}^{2}=c\right\}$ for $c>0$. Very recently, using the techniques introduced in [1, 20], Luo and Wang [21] established the existence of infinitely many couples of solutions $\left\{\left(u_{n}^{b}, \lambda_{n}\right)\right\} \subset$ $\bar{S}_{r}(c) \times \mathbf{R}^{-}$for the following Kirchhoff type problem:

$$
-\left(a+b \int_{\mathbf{R}^{3}}|\nabla u|^{2} d x\right) \Delta u-\lambda u=|u|^{q-2} u, \quad x \in \mathbf{R}^{3},
$$

for each $n \in \mathbf{N}^{+}$and $q \in\left(\frac{14}{3}, 6\right)$. Moreover, they also analyzed the asymptotic behavior of $u_{n}^{b}$ as $b \rightarrow 0^{+}$.

To the best knowledge of ours, little is known about the existence of normalized solutions of Chern-Simons-Schrödinger equations except for [8, 16, 33]. Set

$$
e_{q}(c):=\inf _{u \in S_{r}(c)} I_{q}(u),
$$

where the functional $I_{q}$ is derived from (1.3) given by

$$
I_{q}(u)=\frac{1}{2} \int_{\mathbf{R}^{2}}|\nabla u|^{2}+\frac{1}{2} \int_{\mathbf{R}^{2}} \frac{|u|^{2}}{|x|^{2}}\left(\int_{0}^{|x|} \frac{r}{2} u^{2}(r) d r\right)^{2}-\frac{1}{q} \int_{\mathbf{R}^{2}}|u|^{q}, \quad u \in H_{r}^{1}\left(\mathbf{R}^{2}\right) .
$$

It is standard that the minimizers of $e_{q}(c)$ are exactly critical points of $I_{q}$ restricted to $S_{r}(c)$, and thus normalized solutions of (1.3). By scaling arguments, it is readily seen that $q=4$ is $L^{2}$-critical exponent for (1.10) in the sense that for any $c>0$, $e_{q}(c)>-\infty$ if $q \in(2,4)$ and $e_{q}(c)=-\infty$ if $q>4$.

In [8], Byeon et al. proved that problem (1.10) admits a positive minimizer provided that $c>0$ is sufficiently small whenever $q \in(3,4)$ or $c>0$ is arbitrary whenever $q \in(2,3]$. If $c$ and $q$ satisfy the above assumptions, then Yuan [33] obtained infinitely many distinct pairs of solutions $\left(u_{n}, \lambda_{n}\right) \subset H_{r}^{1}\left(\mathbf{R}^{2}\right) \times \mathbf{R}^{-}$of (1.3) for each $n \in \mathbf{N}^{+}$ via the argument of Krasnoselski genus (see [26]). Furthermore, motivated by [1], the 
author also proved that for $q>4,(1.3)$ admits an unbounded sequence of couples of solutions $\left(u_{n}, \lambda_{n}\right) \subset H_{r}^{1}\left(\mathbf{R}^{2}\right) \times \mathbf{R}^{-}$for $c \in\left(0, c_{0}\right)$ sufficient small.

In [16], Li and Luo considered problem (1.3) with $q \geq 4$. For $q=4$, they showed a sufficient condition for the nonexistence of constraint critical points of $I_{q}$ on $S_{r}(c)$ for certain $c>0$ and obtained infinitely many minimizers of $I_{q}$ on $S_{r}(8 \pi)$. When $q>4$, using a minimax procedure motivated by [1], the authors proved the multiplicity of normalized solutions for (1.3) for $c \in\left(0, \frac{4 \pi}{\sqrt{p-3}}\right)$. Compared with [33], Li and Luo presented a certain constant $c_{0}=\frac{4 \pi}{\sqrt{p-3}}$, which improved the result for the case $q>4$ in [33]. Moreover, the existence of normalized solutions for (1.3) was also considered in [16]. To this end, they used the approach introduced in [24] to construct a suitable submanifold of $S_{r}(c)$, which is defined by a condition which is a combination of the related Nehari functional and Pohozaev identity, i.e.,

$$
\widetilde{V}(c)=\left\{u \in S_{r}(c): Q(u)=0\right\},
$$

where

$$
Q(u)=\int_{\mathbf{R}^{2}}|\nabla u|^{2}+\int_{\mathbf{R}^{2}} \frac{|u|^{2}}{|x|^{2}}\left(\int_{0}^{|x|} \frac{r}{2} u^{2}(r) d r\right)^{2}-\frac{q-2}{q} \int_{\mathbf{R}^{2}}|u|^{q} .
$$

Motivated by all results mentioned previously, our contribution in this paper is to generalize the existence and the multiplicity result of normalized solutions for (1.3) in $[16,33]$ to $(1.4)$. We emphasize that, at least in our knowledge, does not exist in the literature actually available results involving the existence of normalized solutions for (1.4) with general nonlinearities. To state our main results, we give some definitions and nations. Analogous to (1.11), set

$$
V(c)=\left\{u \in S_{r}(c): J_{\kappa}(u)=0\right\}, \quad m(c):=\inf _{u \in V(c)} E_{\kappa}(u)
$$

where

$$
J_{\kappa}(u)=\int_{\mathbf{R}^{2}}|\nabla u|^{2}+\kappa \int_{\mathbf{R}^{2}} \frac{|u|^{2}}{|x|^{2}}\left(\int_{0}^{|x|} \frac{r}{2} u^{2}(r) d r\right)^{2}-\int_{\mathbf{R}^{2}}[f(u) u-2 F(u)] .
$$

In addition, we shall prove that $E_{\kappa}$ has a MP geometry on $S_{r}(c)$ and $m(c)=\gamma(c)$ (see Lemma 2.6).

Definition 1.1. [3, Definition 1.1] Given $c>0$, we say that $E_{\kappa}(u)$ has a MP geometry on $S_{r}(c)$, if there exists $K_{c}>0$ such that

$$
\gamma(c)=\inf _{g \in \Gamma_{c}} \max _{t \in[0,1]} E_{\kappa}(g(t))>\max \left\{\max _{g \in \Gamma_{c}} E_{\kappa}(g(0)), \max _{g \in \Gamma_{c}} E_{\kappa}(g(1))\right\}
$$

holds in the set $\Gamma(c):=\left\{g \in \mathcal{C}\left([0,1], S_{r}(c)\right): g(0) \in A_{K_{c}}\right.$ and $\left.E_{\kappa}(g(1))<0\right\}$, where

$$
A_{K_{c}}=\left\{u \in S_{r}(c):\|\nabla u\|_{2}^{2} \leq K_{c}\right\} .
$$

Our main results are as follows:

Theorem 1.1. Assume that $\left(f_{1}\right)-\left(f_{4}\right)$ hold.

(i) Then for any $c>0$ and $\kappa>0, E_{\kappa}$ has a MP geometry on $S_{r}(c)$.

(ii) Then there exists a certain $c_{*}>0$ such that for any $c \in\left(0, c_{*}\right]$ and $\kappa>0$, there exists a couple of solution $\left(u_{c}, \lambda_{c}\right) \in S_{r}(c) \times \mathbf{R}^{-}$for $(1.4)$ with $E_{\kappa}\left(u_{c}\right)=m(c)$ and $u_{c}$ is nonnegative. In addition, $\left\|\nabla u_{c}\right\|_{2} \rightarrow \infty$ and $\lambda_{c} \rightarrow-\infty$ as $c \rightarrow 0$.

Theorem 1.2. Assume that $\left(f_{1}\right)-\left(f_{5}\right)$ hold. 
(i) Then for any $c \in\left(0, \frac{4 \pi}{\sqrt{p-3}}\right)$ and $\kappa \in(0, p-3)$, problem (1.4) admits an unbounded sequence of couples of solutions $\left(u_{n}, \lambda_{n}\right) \in S_{r}(c) \times \mathbf{R}^{-}$for each $n \in \mathbf{N}^{+}$.

(ii) Then there exists $\kappa_{0}>0$ such that for any $\kappa \in\left(0, \kappa_{0}\right)$ and $c>0$, problem (1.4) admits an unbounded sequence of couples of solutions $\left(u_{n}^{\kappa}, \lambda_{n}^{\kappa}\right) \in S_{r}(c) \times \mathbf{R}^{-}$ for each $n \in \mathbf{N}^{+}$.

Corollary 1.3. Assume that $\left(f_{1}\right)-\left(f_{5}\right)$ hold. Then there exists an unbounded sequence of couples of solutions $\left\{\left(u_{n}, \lambda_{n}\right)\right\} \subset S_{r}(c) \times \mathbf{R}^{-}$for the following equation:

$$
-\Delta u-\lambda u=f(u), \quad \text { in } \mathbf{R}^{2} \text {. }
$$

Motivated by Theorem 1.2 (ii) and Corollary 1.3, we attempt to investigate the convergence property of $u_{n}^{\kappa}$ and $\lambda_{n}^{\kappa}$ found in Theorem 1.2 (ii) as $\kappa \rightarrow 0$. Then we have the following theorem.

Theorem 1.4. Let $\left\{\left(u_{n}^{\kappa}, \lambda_{n}^{\kappa}\right)\right\} \subset S_{r}(c) \times \mathbf{R}^{-}$be found in Theorem 1.2 (ii). Then for any sequence $\left\{\kappa_{m}\right\} \rightarrow 0^{+}$as $m \rightarrow \infty$, there exists a subsequence of $\left\{\kappa_{m}\right\}$, still denoted by $\left\{\kappa_{m}\right\}$, such that for any $n \in \mathbf{N}^{+},\left(u_{n}^{\kappa_{m}}, \lambda_{n}^{\kappa_{m}}\right) \rightarrow\left(u_{n}^{0}, \lambda_{n}^{0}\right)$ as $m \rightarrow \infty$, where $\left\{\left(u_{n}^{0}, \lambda_{n}^{0}\right)\right\} \subset S_{r}(c) \times \mathbf{R}^{-}$is a sequence of couples of solutions for (1.13).

Remark 1.1. It is easy to check that the function

$$
f(s)=\sum_{i=1}^{m}|s|^{p_{i}-2} s \text { for } p_{i}>4, \quad 1 \leq i \leq m,
$$

satisfies $\left(f_{1}\right)-\left(f_{5}\right)$ and $\kappa=1 \in(0, p-3)$ due to $p>4$. Thus, the results of Theorem 1.1 (ii) and Theorem 1.2 (i) generalize and improve ones of Theorems 1.2-1.3 in [16] and Theorem 1.1 (3) in [33]. Moreover, the solutions obtained in Theorem 1.1 are mountain pass type and thus its Morse index is 1 , which is not considered in $[16,33]$.

Remark 1.2. The strict monotonicity of the function $c \mapsto m(c)$ is essential for the proof of Theorem 1.1 (ii), as well as Theorem 1.2 in [16]. To prove the property, [16] gave the restriction that $c \in\left(0,(2 p-4)^{\frac{2-p}{2 p-5}}\right]$ for $p>4$. As described in Lemma 2.8, however, we obtained a larger range that $c \in\left(0, \frac{4 \pi}{\sqrt{2 p-4}}\right]$ for $p>4$. In particular, due to the general nonlinearity $f$, it does not seems possible to deduce the strict monotonicity of $c \mapsto m(c)$. In addition, it is worth pointing out that in [16, Theorem 1.2] $\lambda_{c}$ should be negative due to $c^{*}:=(2 p-4)^{\frac{2-p}{2 p-5}}<\frac{4 \pi}{\sqrt{p-3}}$.

Remark 1.3. The idea of Theorem 1.4 comes from [17], which studied the existence and asymptotic behavior of least energy sign-changing solutions for (1.4) with $f(u)=|u|^{q-2} u(q>6)$. But in [17] there is no information about the $L^{2}$-norm of the solutions. Hence, Theorems 1.1-1.3 can be also regarded as a complement of the main results in [17]. In addition, the results of Theorem 1.2 (ii) and Theorem 1.4 are new, even for problem (1.3).

Obviously, the conditions $\left(f_{1}\right)-\left(f_{5}\right)$ imply that the functional $E_{\kappa}$ is no longer bounded form below on $S_{r}(c)$. Therefore, the minimization method on $S_{r}(c)$ used in [8] does not work. To prove Theorem 1.1, we construct a submanifold $V(c)$ of $S_{r}(c)$, on which $E_{\kappa}$ is bounded from below and then we prove that the minimum of $E_{\kappa}$ on $V(c)$ is attained. This approach is motivated by [16]. However, we have to overcome three main difficulties. Firstly, different from [16], it does not seem possible to prove the coercivity of $E_{\kappa}$ on $V(c)$. Therefore, the first difficulty is to verify the 
boundedness of the minimizing sequence $\left\{u_{n}\right\} \subset V(c)$ of $E_{\kappa}$. We use the assumption $\left(H_{2}\right)$ in [18] to deal with such a difficulty. But the presence of the nonlocal term

$$
\int_{\mathbf{R}^{2}} \frac{|u|^{2}}{|x|^{2}}\left(\int_{0}^{|x|} \frac{r}{2} u^{2}(r) d r\right)^{2}
$$

in $E_{\kappa}$ would require extra efforts to be treated. Secondly, because it is not assumed that $f$ is differentiable, it is difficult to prove that $\left.E_{\kappa}\right|_{V(c)}$ is a natural constraint of $\left.E_{\kappa}\right|_{S_{r}(c)}$, which is not derived through the use of Lagrange multiplier theorem adopted in [16]. Instead, we use the quantitative deformation lemma on $S_{r}(c)$, which was introduced by [3]. Finally, although the workspace is $H_{r}^{1}\left(\mathbf{R}^{2}\right)$, the difficulty that the weak limit $\bar{u} \in H_{r}^{1}\left(\mathbf{R}^{2}\right)$ of $\left\{u_{n}\right\}$ does not necessarily lie in $V(c)$ still exists as observed in [16]. Because our minimization problem is constrained on a submanifold of $S_{r}(c)$ but not on that of $H_{r}^{1}\left(\mathbf{R}^{2}\right)$, it is more difficult to prove that $V(c)$ is weakly closed. To circumvent this obstacle, we prove the monotonicity of the function $c \mapsto m(c)$ and borrow some ideas from [27].

For Theorem 1.2, Since $E_{\kappa}$ is unbounded from below on $S_{r}(c)$, the genus of the sublevel set

$$
E_{\kappa}^{d}:\left\{u \in S_{r}(c): E_{\kappa}(u) \leq d\right\}
$$

is always infinite. This shows that the classical argument based on the Krasnoselski genus seems not applicable to our case. To prove Theorem 1.2, we mainly follow the strategy of [1] to construct a special (PS) sequence at high energy level $\gamma_{n}(c)$ for each fixed $n \in \mathbf{N}^{+}$and prove its boundedness and compactness. Compared with [1], we get rid of the condition $0<\alpha F(s) \leq f(s) s$ in $\left(H_{2}\right)$, which seems essential to ensure the boundedness of the (PS) sequence in [1], as well as in [18]. Instead, we assume that $c \in\left(0, \frac{4 \pi}{\sqrt{p-3}}\right)$ and $\kappa \in(0, p-3)$ to prove the boundedness of (PS) sequence. In addition, the restriction that $c \in\left(0, \frac{4 \pi}{\sqrt{p-3}}\right)$ originates in the need to show that the associated Lagrange multiplier $\lambda_{c}$ are strictly negative. This property is used to recover the compactness of (PS) sequence.

Remark 1.4. Let $N=2$ in $\left(H_{2}\right)$. Obviously, $\left(H_{1}\right)$ and $\left(H_{2}\right)$ imply $\left(f_{1}\right)-\left(f_{3}\right)$, $\left(f_{5}\right)$. It is easy to check that (1.14) also satisfies $\left(H_{1}\right)$ and $\left(H_{2}\right)$. However, the following functions

and

$$
f(s)=4 s^{3} \ln \left(1+s^{2}\right)+\frac{2 s^{5}}{1+s^{2}}
$$

$$
f(s)=s^{3}+|s|^{p-2} s, \quad p>4,
$$

satisfy $\left(f_{1}\right)-\left(f_{4}\right)$, but do not satisfy $\left(H_{2}\right)$.

The remainder of this paper is organized as follows. In Section 2, we give the proof of Theorem 1.1. Sections 3 is devoted to dealing with the proof of Theorems 1.2 and 1.4 and Corollary 1.3.

Notation. Throughout the article, we let $u^{t}(x):=t u(t x)$ for $t>0$. Denote by $C, C_{k}, k=1,2, \cdots$ various positive constants whose exact value is inessential. For $r>0$ and $y \in \mathbf{R}^{2}$, we denote by $B_{r}(y)$ the open ball in $\mathbf{R}^{2}$ with center $y$ and radius $r$. We denote by $\rightarrow(\rightarrow)$ the strong (weak) convergence. We consider the Hilbert space $H^{1}\left(\mathbf{R}^{2}\right)$ with the norm

$$
\|u\|=\left(\int_{\mathbf{R}^{2}}\left(|\nabla u|^{2}+u^{2}\right)\right)^{\frac{1}{2}} .
$$


$H_{r}^{1}\left(\mathbf{R}^{2}\right)$ denotes the set of the radially symmetric functions in $H^{1}\left(\mathbf{R}^{2}\right)$. Denote the standard norm of $L^{p}\left(\mathbf{R}^{2}\right)(1 \leq p<\infty)$ by $\|u\|_{p}$. Recall that a sequence $\left\{u_{n}\right\} \subset$ $H^{1}\left(\mathbf{R}^{2}\right)$ is said to be a PS sequence for $E$ if

$$
E\left(u_{n}\right) \text { is bounded and } E^{\prime}\left(u_{n}\right) \rightarrow 0 \text { as } n \rightarrow \infty .
$$

We say $E$ satisfies the PS condition if any PS sequence contains a convergent subsequence.

\section{Proof of Theorem 1.1}

In this section, without loss of generality, let $\kappa=1$. For simplicity, denote by $E(u)$ and $J(u)$ the functionals $E_{1}(u)$ and $J_{1}(u)$, respectively. Let

$$
A(u):=\int_{\mathbf{R}^{2}} \frac{|u|^{2}}{|x|^{2}}\left(\int_{0}^{|x|} \frac{r}{2}|u|^{2} d r\right)^{2} .
$$

From now on we assume that $\left(f_{1}\right)-\left(f_{4}\right)$ hold. Similar to the discussion of Proposition 2.3 and Lemma 3.2 in [8], we can get the following conclusions. then

Lemma 2.1. $A \in \mathcal{C}^{1}\left(H_{r}^{1}\left(\mathbf{R}^{2}\right), \mathbf{R}\right)$. Moreover, if $u_{n} \rightarrow u$ in $H_{r}^{1}\left(\mathbf{R}^{2}\right)$, as $n \rightarrow \infty$, $\lim _{n \rightarrow \infty} A\left(u_{n}\right)=A(u), \lim _{n \rightarrow \infty}\left\langle A^{\prime}\left(u_{n}\right), u_{n}\right\rangle=\left\langle A^{\prime}(u), u\right\rangle$ and $\lim _{n \rightarrow \infty}\left\langle A^{\prime}\left(u_{n}\right), \varphi\right\rangle=\left\langle A^{\prime}(u), \varphi\right\rangle$, for any $\varphi \in E$.

Lemma 2.2. Let $b, c$ and $d$ be real constants and $u \in H_{r}^{1}\left(\mathbf{R}^{2}\right)$ be a weak solution of the equation:

$$
\Delta u+b u+c\left(\frac{h^{2}(|x|)}{|x|^{2}}+\int_{|x|}^{\infty} \frac{h(s)}{s} u^{2}(s) d s\right) u+d f(u)=0, \quad x \in \mathbf{R}^{2},
$$

where $h(s)=\frac{1}{2} \int_{0}^{s} r u^{2}(r) d r$. Then there holds the following Pohozaev identity

$$
b \int_{\mathbf{R}^{2}}|u|^{2}+2 c A(u)+2 d \int_{\mathbf{R}^{2}} F(u)=0 .
$$

To estimate the quantity $A(u)$, we present the following lemma.

Lemma 2.3. [16, Lemma 2.3] For $u \in H_{r}^{1}\left(\mathbf{R}^{2}\right)$, the following inequality holds

$$
A(u) \leq \frac{1}{16 \pi^{2}}\|u\|_{2}^{4}\|\nabla u\|_{2}^{2}
$$

Now we give some preliminary lemmas to show some properties of $V(c)$.

Lemma 2.4. For each $u \in S_{r}(c)$,

$$
E(u) \geq E\left(u^{t}\right)+\frac{1-t^{2}}{2} J(u), \quad \forall t \geq 0 .
$$

Proof. We first claim that the following inequality holds:

$$
\frac{1-t^{2}}{2} f(\tau) \tau+\left(t^{2}-2\right) F(\tau)+\frac{1}{t^{2}} F(t \tau) \geq 0, \quad \forall t>0, \tau \in \mathbf{R} .
$$

Indeed, it is evident that (2.2) holds for $\tau=0$. For $\tau \neq 0$, we denote

$$
g(t)=\frac{1-t^{2}}{2} f(\tau) \tau+\left(t^{2}-2\right) F(\tau)+\frac{1}{t^{2}} F(t \tau), \quad \forall t>0
$$


After direct calculations, we see that

$$
g^{\prime}(t)=t|\tau|^{4}\left[\frac{f(t \tau) t \tau-2 F(t \tau)}{(t|\tau|)^{4}}-\frac{f(\tau) \tau-2 F(\tau)}{|\tau|^{4}}\right] .
$$

This relation and $\left(f_{4}\right)$ mean that $g^{\prime}(t)>0$ for $t>1$ and $g^{\prime}(t)<0$ for $0<t<1$, that is,

$$
g(t)>g(1)=0, \quad \text { for } t \neq 1 .
$$

Therefore, (2.2) follows. Note that

$$
E\left(u^{t}\right)=\frac{t^{2}}{2} \int_{\mathbf{R}^{2}}|\nabla u|^{2}+\frac{t^{2}}{2} A(u)-\frac{1}{t^{2}} \int_{\mathbf{R}^{2}} F(t u) .
$$

Then, from (2.2), (2.4) and (1.12), we get that

$$
\begin{aligned}
E(u)-E\left(u^{t}\right) & =\frac{1-t^{2}}{2} \int_{\mathbf{R}^{2}}|\nabla u|^{2}+\frac{1-t^{2}}{2} A(u)+\int_{\mathbf{R}^{2}}\left(\frac{1}{t^{2}} F(t u)-F(u)\right) \\
& =\frac{1-t^{2}}{2} J(u)+\int_{\mathbf{R}^{2}}\left[\frac{1-t^{2}}{2} f(u) u+\left(t^{2}-2\right) F(u)+\frac{1}{t^{2}} F(t u)\right] \\
& \geq \frac{1-t^{2}}{2} J(u),
\end{aligned}
$$

and this proves (2.1).

Lemma 2.5. For each $u \in S_{r}(c)$, there exists a unique $\tilde{t}=t(u)>0$ such that $u^{\tilde{t}} \in V(c)$. Moreover, $E\left(u^{\tilde{t}}\right)=\max _{t \geq 0} E\left(u^{t}\right)$.

Proof. Consider a function $\Phi(t):=E\left(u^{t}\right)$ on $[0, \infty)$. By $\left(f_{1}\right)-\left(f_{3}\right)$ and $(2.4)$, it is easy to check that $\Phi(0)=0, \Phi(t)>0$ for $t>0$ small and $\Phi(t)<0$ for $t$ large. Hence, $\max _{t \geq 0} \Phi(t)$ is achieved at $\tilde{t}=t(u)>0$ and then $\Phi^{\prime}(\tilde{t})=0$, that is,

$$
\tilde{t}^{2} \int_{\mathbf{R}^{2}}|\nabla u|^{2}+\tilde{t}^{2} A(u)-\frac{1}{\tilde{t}^{2}} \int_{\mathbf{R}^{2}}[f(\tilde{t} u) \tilde{t} u-2 F(\tilde{t} u)]=0 .
$$

This shows that $J\left(u^{\tilde{t}}\right)=0$ and $u^{\tilde{t}} \in V(c)$.

Next we prove that $\tilde{t}$ is unique for any $u \in S_{r}(c)$. Let $t_{1}, t_{2}>0$ be such that $u^{t_{1}}, u^{t_{2}} \in V(c)$ and $t_{2}=a t_{1}$. Then $J\left(u^{t_{1}}\right)=J\left(u^{t_{2}}\right)=0$. From (2.5), one has

$$
E\left(u^{t_{1}}\right)=E\left(u^{t_{2}}\right)+\int_{\mathbf{R}^{2}}\left[\frac{1-a^{2}}{2} f(u) u+\left(a^{2}-2\right) F(u)+\frac{1}{a^{2}} F(a u)\right]
$$

and

$$
E\left(u^{t_{2}}\right)=E\left(u^{t_{1}}\right)+\int_{\mathbf{R}^{2}}\left[\frac{1-a^{-2}}{2} f(u) u+\left(a^{-2}-2\right) F(u)+a^{2} F\left(a^{-1} u\right)\right],
$$

which together with (2.3) imply that $a=1$, i.e., $t_{1}=t_{2}$. In addition, it is readily checked that $E\left(u^{\tilde{t}}\right)=\max _{t \geq 0} E\left(u^{t}\right)$.

Thanks to Lemma 2.5, we get that

$$
m(c)=\inf _{u \in S_{r}(c)} \max _{t \geq 0} E\left(u^{t}\right)>0 .
$$

Let $t \rightarrow 0$ in (2.2), then we get from $\left(f_{1}\right)$ and (2.3) that

$$
f(\tau) \tau-4 F(\tau)>0, \quad \forall \tau \in \mathbf{R} \backslash\{0\} .
$$

By $\left(f_{2}\right)$ and $(2.7)$, one has

$$
4 F(t)<f(t) t \leq p F(t), \forall t \in \mathbf{R} \backslash\{0\},
$$


which implies that for all $t \in \mathbf{R}$,

$$
\begin{cases}|s|^{p} F(t) \leq F(t s) \leq|s|^{4} F(t), & \text { if }|s| \leq 1 ; \\ |s|^{4} F(t) \leq F(t s) \leq|s|^{p} F(t), & \text { if }|s| \geq 1 ;\end{cases}
$$

and $F(t)>0$ for $t \neq 0$. In particular, taking $t=1$ in $(2.9)$, we deduce from $\left(f_{1}\right)$ that for any $\varepsilon>0$, there exists $C_{\varepsilon}>0$ such that

$$
|F(s)| \leq \varepsilon|s|^{2}+C_{\varepsilon}|s|^{p} \text { for all } s \in \mathbf{R} \text {. }
$$

In what follows we shall need the following Gagliardo-Nirenberg type result (see [30]): let $q \geq 2$ and $u \in H_{r}^{1}\left(\mathbf{R}^{2}\right)$, then

$$
\|u\|_{q}^{q} \leq C(q)\|\nabla u\|_{2}^{q-2}\|u\|_{2}^{2}
$$

with equality holds for $u=W_{q}$, where $C(q)=\frac{q}{2}\left\|W_{q}\right\|_{2}^{2-q}$ and, up to translations, $W_{q}$ is the unique ground state solution of

$$
-\frac{q-2}{2} \Delta W+W=|W|^{q-2} W, \quad x \in \mathbf{R}^{2} .
$$

Lemma 2.6. For $c>0, E$ has a $M P$ geometry on $S_{r}(c)$. Moreover, $m(c)=\gamma(c)$, where $\gamma(c)$ is defined in Definition 1.1.

Proof. It follows from (2.7) that for any $u \in S_{r}(c)$,

$$
E(u)-\frac{1}{2} J(u)=\frac{1}{2} \int_{\mathbf{R}^{2}}[f(u) u-4 F(u)]>0 .
$$

We next show that there exist $0<k_{1}<k_{2}$ such that

$$
0<\alpha_{k_{1}}:=\sup _{y \in A_{k_{1}}} E(u)<\nu_{k_{2}}:=\inf _{u \in \partial A_{k_{2}}} E(u),
$$

where $A_{k}$ is introduced in Definition 1.1. Note that by (2.9), (2.11) and Lemma 2.3, we see that

$$
\begin{aligned}
|E(u)| & \leq \frac{1}{2}\|\nabla u\|_{2}^{2}+\frac{1}{2} A(u)+F(1)\left(\|u\|_{4}^{4}+\|u\|_{p}^{p}\right) \\
& \leq \frac{1}{2}\|\nabla u\|_{2}^{2}+C_{1}\|\nabla u\|_{2}^{2}+C_{2}\|\nabla u\|_{2}^{2}+C_{3}\|\nabla u\|_{2}^{p-2} .
\end{aligned}
$$

In particular, $\alpha_{k_{1}} \rightarrow 0^{+}$as $k_{1} \rightarrow 0^{+}$. On the other hand, from (2.10), it follows that

$$
\begin{aligned}
E(u) & =\frac{1}{2}\|\nabla u\|_{2}^{2}+\frac{1}{2} A(u)-\int_{\mathbf{R}^{2}} F(u) \geq \frac{1}{2}\|\nabla u\|_{2}^{2}-\varepsilon\|u\|_{2}^{2}-C_{\varepsilon}\|u\|_{p}^{p} \\
& \geq \frac{1}{2}\|\nabla u\|_{2}^{2}-\varepsilon c-C_{2}\|\nabla u\|_{2}^{p-2} .
\end{aligned}
$$

Thus, since $p>4$ and $\varepsilon$ is arbitrary, we have $\nu_{k_{2}} \geq \frac{1}{8} k_{2}$ for $k_{2}>0$ small. These two observations imply that (2.13) holds. We now fix $0<k_{1}<k_{2}$ as in (2.13). From $\left(f_{3}\right)$ and $(2.4)$, it is readily checked that

$$
\left\|\nabla u^{t}\right\| \rightarrow \infty \quad \text { and } \quad E\left(u^{t}\right) \rightarrow-\infty \quad \text { as } t \rightarrow \infty .
$$

Thus $\Gamma_{c} \neq \emptyset$. Then form the definition of $\gamma(c)$, we get that $\gamma(c) \geq \nu_{k_{2}}>0$.

For any $u \in V(c)$, from $\left\|\nabla u^{t}\right\|_{2}^{2}=t^{2}\|\nabla u\|_{2}^{2}$ and (2.16), we deduce that there exist $t_{1}>0$ small and $t_{2}>0$ large such that $u^{t_{1}} \in A_{k_{1}}$ and $E\left(u^{t_{2}}\right)<0$. So, if we define

$$
g(\tau)=u^{(1-\tau) t_{1}+\tau t_{2}}, \quad \text { for } \tau \in[0,1]
$$


then we obtain a path in $\Gamma_{c}$. Using $(2.6)$,

$$
\gamma(c) \leq \max _{\tau \in[0,1]} E(g(\tau)) \leq \max _{\tau \geq 0} E\left(u^{\tau}\right)=E(u)
$$

and thus $\gamma(c) \leq m(c)$. On the other hand, from $\left(f_{2}\right),(1.12)$ and (2.10), it follows that

$$
\begin{aligned}
J(u) & \geq\|\nabla u\|_{2}^{2}+A(u)-(p-2) \int_{\mathbf{R}^{2}} F(u) \\
& \geq\|\nabla u\|_{2}^{2}-\varepsilon(p-2)\|u\|_{2}^{2}-C_{\varepsilon}\|u\|_{p}^{p} \\
& \geq\|\nabla u\|_{2}^{2}-\varepsilon(p-2) c-C_{2}\|\nabla u\|_{2}^{p-2} .
\end{aligned}
$$

The fact that $p>4$ and $\varepsilon$ is arbitrary ensures that $J(u)>0$ for $\|\nabla u\|_{2}^{2}<k_{1}$. Then (2.12) implies that $J(g([0,1])) \cap V(c) \neq \emptyset$ for any $g \in \Gamma(c)$. Hence $\gamma(c) \geq m(c)$. The proof is completed.

Lemma 2.7. For $c>0, E$ is bounded from below on $V(c)$. Moreover for any $u \in V(c)$, there exists a constant $\rho>0$ such that $\|\nabla u\|_{2}^{2} \geq \rho$.

Proof. For any $u \in V(c)$, it follows from Lemma 2.6 that that $m(c)=\gamma(c) \geq$ $\nu_{k_{2}}>0$. This together with (2.14) implies that $\|\nabla u\|_{2}^{2} \geq \rho$.

Lemma 2.8. For any $c \in\left(0, \frac{4 \pi}{\sqrt{2 p-4}}\right]$, the function $c \mapsto m(c)$ is non-increasing, where $p$ is given in $\left(f_{2}\right)$.

Proof. For any $0<c_{1}<c_{2} \leq \frac{4 \pi}{\sqrt{2 p-4}}$ and $p>4$, from Lemma 2.5 and (2.4), it follows that there exists $\left\{u_{n}\right\} \subset V\left(c_{1}\right)$ such that

$$
E\left(u_{n}\right)=\max _{t \geq 0} E\left(u_{n}^{t}\right) \leq m\left(c_{1}\right)+\frac{1}{n} .
$$

We first claim that for any $0<c_{1}<c_{2} \leq \frac{4 \pi}{\sqrt{2 p-4}}$ and $p>4$, then

$$
c_{2}^{\frac{1}{p-2}}\left(c_{2}^{2}-c_{1}^{2}\right) \leq 16 \pi^{2}\left(c_{2}^{\frac{1}{p-2}}-c_{1}^{\frac{1}{p-2}}\right) .
$$

Indeed, it is sufficient to prove that

$$
c_{2}^{2} \leq 16 \pi^{2} \frac{1-a^{1 /(2-p)}}{1-a^{-2}}, \quad \text { where } a:=\frac{c_{2}}{c_{1}}>1 .
$$

Set the function $f(x)=\frac{1-x}{1-x^{2 p-4}}, x \in(0,1)$. After direct calculations, we see that

$$
\begin{aligned}
& f^{\prime}(x)=\frac{1}{\left(1-x^{2 p-4}\right)^{2}}\left[(5-2 p) x^{2 p-4}+(2 p-4) x^{2 p-5}-1\right]:=\frac{1}{\left(1-x^{2 p-4}\right)^{2}} g(x) ; \\
& g^{\prime}(x)=(2 p-5)(2 p-4) x^{2 p-6}(1-x) .
\end{aligned}
$$

From the expression of $g^{\prime}(x)$ and $p>4$, we know that $g(x) \leq g(1)=0$ and thus $f^{\prime}(x)<0$ for $x \in(0,1)$. Then we have that

$$
f(x) \geq \lim _{x \rightarrow 1^{-}} \frac{1-x}{1-x^{2 p-4}}=\frac{1}{2 p-4}, \quad \forall x \in(0,1) .
$$

This proves the assertion. Then it is easy to check that for $n \in \mathbf{N}^{+}$,

$$
\frac{c_{1}^{2}}{16 \pi^{2}}\left(a^{2}-a^{\frac{1}{p-2}}\right)\left\|\nabla u_{n}\right\|_{2}^{2} \leq\left(a^{\frac{1}{p-2}}-1\right)\|\nabla u\|_{2}^{2}
$$

which together with Lemma 2.3 shows that

$$
\left\|\nabla u_{n}\right\|_{2}^{2}+a^{2} A\left(u_{n}\right) \leq a^{\frac{1}{p-2}}\left(\left\|\nabla u_{n}\right\|_{2}^{2}+A\left(u_{n}\right)\right), \forall n \in \mathbf{N}^{+} .
$$


Let $v_{n}=u_{n}\left(a^{-\frac{1}{2}} x\right)$, then $\left\|v_{n}\right\|_{2}^{2}=c_{2}$. Note that $\left(f_{2}\right)$ implies that $\frac{F(\tau)}{|\tau|^{p-1} \tau}$ is nonincreasing in $\mathbf{R} \backslash\{0\}$. Therefore, for any $\tau \in \mathbf{R} \backslash\{0\}$,

$$
\begin{aligned}
0 & \leq\left(\frac{F(\tau)}{|\tau|^{p}}-\frac{F\left(a^{\frac{1}{2(p-2)}} \tau\right)}{\left(a^{\frac{1}{2(p-2)}}|\tau|\right)^{p}}\right)|\tau|^{p} \\
& =F(\tau)-a^{-\frac{p}{2(p-2)}} F\left(a^{\frac{1}{2(p-2)}} \tau\right) \leq a F(\tau)-a^{\frac{p-4}{2(p-2)}} F\left(a^{\frac{1}{2(p-2)}} \tau\right) \\
& =a F(\tau)-a^{-\frac{1}{(p-2)}} F\left(a^{\frac{1}{2(p-2)}} \tau\right)-a^{-\frac{1}{(p-2)}}\left(a^{\frac{p-2}{2(p-2)}}-1\right) F\left(a^{\frac{1}{2(p-2)}} \tau\right) \\
& <a F(\tau)-a^{-\frac{1}{(p-2)}} F\left(a^{\frac{1}{2(p-2)}} \tau\right),
\end{aligned}
$$

since $a>1$ and $p>4$. In virtue of Lemma 2.5, there exists $t_{n}>0$ such that $v_{n}^{t_{n}} \in V\left(c_{2}\right)$. Then by (2.19) and (2.20), we see that

$$
\begin{aligned}
m\left(c_{2}\right) & \leq E\left(v_{n}^{t_{n}}\right)=\frac{t_{n}^{2}}{2}\left(\left\|\nabla u_{n}\right\|_{2}^{2}+a^{2} A\left(u_{n}\right)\right)-\frac{a}{t_{n}^{2}} \int_{\mathbf{R}^{2}} F\left(t_{n} u_{n}\right) \\
& \leq \frac{1}{2} t_{n}^{2} a^{\frac{1}{(p-2)}}\left(\left\|\nabla u_{n}\right\|_{2}^{2}+A\left(u_{n}\right)\right)-\frac{a}{t_{n}^{2}} \int_{\mathbf{R}^{2}} F\left(t_{n} u_{n}\right) \\
& \leq E\left(u_{n}^{a^{\frac{1}{2(p-2)}} t_{n}}\right)+\frac{a^{-\frac{1}{(p-2)}}}{t_{n}^{2}} \int_{\mathbf{R}^{2}} F\left(a^{\frac{1}{2(p-2)}} t_{n} u_{n}\right)-\frac{a}{t_{n}^{2}} \int_{\mathbf{R}^{2}} F\left(t_{n} u_{n}\right) \\
& <m\left(c_{1}\right)+\frac{1}{n}
\end{aligned}
$$

which shows that $m\left(c_{2}\right) \leq m\left(c_{1}\right)$ by letting $n \rightarrow \infty$.

Lemma 2.9. There exists $c_{*}>0$ such that for any $c \in\left(0, c_{*}\right], m(c)$ is achieved.

Proof. It follows from Lemma 2.7 that $m(c) \geq \nu_{k_{2}}>0$. Take

$$
c_{*}:=\min \left\{\frac{4 \pi}{\sqrt{2 p-4}},(8 C(4) F(1))^{-1},(C(p) F(1))^{-1} 8^{\frac{2-p}{2}}(m(c))^{\frac{4-p}{2}}\right\},
$$

where $C(q)$ and $p$ is given in (2.11) and $\left(f_{2}\right)$, respectively. Let $\left\{u_{n}\right\} \subset V(c)$ be such that $E\left(u_{n}\right) \rightarrow m(c)$. Now we show that $\left\{\left\|\nabla u_{n}\right\|_{2}\right\}$ is bounded. Suppose arguing by contradiction $\left\|\nabla u_{n}\right\|_{2} \rightarrow \infty$. Let $t_{n}=\frac{\sqrt{8 m(c)}}{\left\|\nabla u_{n}\right\|_{2}}$. Then from (2.1), (2.4), (2.9), (2.11) and $J\left(u_{n}\right)=0$, we deduce

$$
\begin{aligned}
m(c)+o(1) & =E\left(u_{n}\right) \geq E\left(\left(u_{n}\right)^{t_{n}}\right) \\
& =\frac{t_{n}^{2}}{2}\left\|\nabla u_{n}\right\|_{2}^{2}+\frac{t_{n}^{2}}{2} A\left(u_{n}\right)-\frac{1}{t_{n}^{2}} \int_{\mathbf{R}^{2}} F\left(t_{n} u_{n}\right) \\
& \geq \frac{t_{n}^{2}}{2}\left\|\nabla u_{n}\right\|_{2}^{2}-F(1) \int_{\mathbf{R}^{2}}\left(t_{n}^{2}\left|u_{n}\right|^{4}+t_{n}^{p-2}\left|u_{n}\right|^{p}\right) \\
& \geq \frac{t_{n}^{2}}{2}\left\|\nabla u_{n}\right\|_{2}^{2}-c F(1) C(4) t_{n}^{2}\left\|\nabla u_{n}\right\|_{2}^{2}+c F(1) C(p) t_{n}^{p-2}\left\|\nabla u_{n}\right\|_{2}^{p-2} \\
& \geq 4 m(c)-c F(1)\left(8 m(c) C(4)+C(p)(8 m(c))^{(p-2) / 2}\right) \geq 2 m(c),
\end{aligned}
$$

which contradicts $m(c)>0$. Thus, $\left\{\left\|\nabla u_{n}\right\|_{2}\right\}$ is bounded and then $\left\{u_{n}\right\}$ is also bounded in $H_{r}^{1}\left(\mathbf{R}^{2}\right)$. Then, there exists $u \neq 0$ such that, taking a subsequence if 
necessary,

$$
\begin{cases}u_{n} \rightarrow u & \text { in } H_{r}^{1}\left(\mathbf{R}^{2}\right), \\ u_{n} \rightarrow u & \text { in } L^{q}\left(\mathbf{R}^{2}\right), \\ u_{n} \rightarrow u & \text { a.e. in } \mathbf{R}^{2},\end{cases}
$$

for $q \in(2, \infty)$, and thus by (2.7) and (2.10), we have

$$
\int_{\mathbf{R}^{2}} f\left(u_{n}\right) u_{n} \rightarrow f(u) u \text { and } \int_{\mathbf{R}^{2}} F\left(u_{n}\right) \rightarrow F(u) .
$$

Otherwise, $u=0$. Then it follows from (1.12) and (2.23) that $\left\|\nabla u_{n}\right\|_{2} \rightarrow 0$ and $A\left(u_{n}\right) \rightarrow 0$, which and (2.23) imply $E\left(u_{n}\right) \rightarrow 0$, that is, $m(c)=0$. This is a contradiction. Next we suppose that $\|u\|_{2}^{2}=\bar{c} \in(0, c]$. Then from (1.12), (2.23), Lemma 2.1 and weak lower semi-continuity of norm, we get

$$
J(u) \leq \liminf _{n \rightarrow \infty} J\left(u_{n}\right)=0 .
$$

In virtue of Lemma 2.5, there exists $t_{u}>0$ such that $u^{t_{u}} \in V(\bar{c})$. By (2.1), (2.12), (2.23)-(2.24) and Lemma 2.8, one has

$$
\begin{aligned}
m(\bar{c}) & \geq m(c)=\lim _{n \rightarrow \infty}\left(E\left(u_{n}\right)-\frac{1}{2} J\left(u_{n}\right)\right) \\
& =\frac{1}{2} \int_{\mathbf{R}^{2}}(f(u) u-4 F(u))=E(u)-\frac{1}{2} J(u) \geq E\left(u^{t_{u}}\right)-\frac{t_{u}^{2}}{2} J(u) \\
& \geq m(\bar{c})-\frac{t_{u}^{2}}{2} J(u) .
\end{aligned}
$$

This shows that $J(u)=0$ and $m(c)=m(\bar{c})$. To this end, we only show that $\bar{c}=c$. Arguing indirectly, suppose that $\bar{c}<c$. Let $v_{n}:=u_{n}-u$, then by the Brezis-Lieb Lemma [31, Lemma 1.32],

$$
\left\|v_{n}\right\|_{2}^{2}=c-\bar{c}+o_{n}(1)
$$

We may assume that for large $n$, there exist $t_{n}>0$ and $\beta_{n} \geq \frac{c-\bar{c}}{2}$ such that $v_{n}^{t_{n}} \in$ $V\left(\beta_{n}\right)$. Using the previous argument in $(2.25)$, for large $n$, one has

$$
\begin{aligned}
o_{n}(1) & =E\left(v_{n}\right)-\frac{1}{2} J\left(v_{n}\right) \geq E\left(v_{n}^{t_{n}}\right)-\frac{t_{n}^{2}}{2} J\left(v_{n}\right) \\
& \geq m\left(\beta_{n}\right)+o_{n}(1) \geq m(c)+o_{n}(1) .
\end{aligned}
$$

This is a contradiction and then the proof is complete.

The above lemma shows that the set

$$
M(c):=\left\{u_{c} \in V(c): E\left(u_{c}\right)=\inf _{u \in V(c)} E(u)\right\}
$$

is not empty.

Lemma 2.10. For each $u_{c} \in M(c)$, there exists a $\lambda_{c} \in \mathbf{R}$ such that $\left(u_{c}, \lambda_{c}\right) \in$ $H_{r}^{1}\left(\mathbf{R}^{2}\right) \times \mathbf{R}$ solves (1.4).

Proof. From Lagrange multiplier theorem, to prove the lemma, it suffices to show that any $u_{c} \in M(c)$ is a critical point of $\left.E\right|_{S_{r}(c)}$. The idea of the proof comes from Lemma 6.1 in [3]. We give a detailed proof here for readers' convenience.

Let $u_{c} \in M(c)$ and suppose, by contradiction, that $\left.E^{\prime}\right|_{S_{r}(c)}\left(u_{c}\right) \neq 0$. Then by the continuity of $E^{\prime}$, there exist $\delta>0$ and $\varrho>0$ such that

$$
v \in B_{u_{c}}(3 \delta) \Rightarrow\left\|\left.E^{\prime}\right|_{S_{r}(c)}(v)\right\|_{H_{r}^{-1}\left(\mathbf{R}^{2}\right)} \geq \varrho
$$


where $B_{u_{c}}(\delta):=\left\{v \in S_{r}(c):\left\|v-u_{c}\right\| \leq \delta\right\}$.

Let $\varepsilon:=\left\{\frac{m(c)}{4}, \frac{\varrho \delta}{8}\right\}$, then [3, Lemma 6.1] yields a deformation $\eta \in C([0,1] \times$ $\left.S_{r}(c), S_{r}(c)\right)$ such that

(i) $\eta(1, v)=v$ if $E(u)<m(c)-2 \varepsilon$ or $E(u)>m(c)+2 \varepsilon$;

(ii) $\eta\left(1, E^{m(c)+\varepsilon} \cap B_{u_{c}}(\delta)\right) \subset E^{m(c)-\varepsilon}$, where $E^{d}:=\left\{u \in S_{r}(c): E(u) \leq d\right\}$;

(iii) $E(\eta(1, v)) \leq E(v), \forall v \in S_{r}(c)$.

Now let $g \in \Gamma(c)$ be the path constructed in (2.17) by choosing $u=u_{c} \in V(c)$. By the proof of Lemma 2.6, we can assume without restriction that $\sup _{u \in A_{K_{c}}} E(u)<$ $\gamma(c) / 2$. This and (i) show that $\eta(1, g(\tau)) \in \Gamma(c)$. Note that since $E(g(\tau)) \leq E\left(u_{c}\right)=$ $m(c)=\gamma(c)$ for all $\tau \in[0,1]$, one of the following three cases must occur:

(1) If $g(\tau) \in S_{r}(c) \backslash B_{u_{c}}(\delta)$, then by (iii) and Lemma 2.2,

$$
E(\eta(1, g(\tau))) \leq E\left(g(\tau)<E\left(u_{c}\right)=\gamma(c) .\right.
$$

(2) If $g(\tau) \in E^{\gamma(c)-\varepsilon}$, then using (iii),

$$
E(\eta(1, g(\tau))) \leq E(g(\tau) \leq \gamma(c)-\varepsilon .
$$

(3) If $g(\tau) \in E^{-1}([m(c)-\varepsilon, m(c)+\varepsilon]) \cap B_{u_{c}}(\delta)$, then by (ii),

$$
E(\eta(1, g(\tau))) \leq \gamma(c)-\varepsilon .
$$

Thus we have that

$$
\max _{\tau \in[0,1]} E(\eta(1, g(\tau)))<\gamma(c),
$$

which contradicts the definition of $\gamma(c)$.

Lemma 2.11. If $u_{c} \in H^{1}\left(\mathbf{R}^{2}\right)$ is a weak solution of $(1.4)$, then $J\left(u_{c}\right)=0$. Furthermore, if $\lambda \geq 0$, then the only solution of (1.4) fulfilling $\left\|u_{c}\right\|_{2}^{2}<\frac{4 \pi}{\sqrt{p-3}}$ is null function.

Proof. It follows from Lemma 2.2 that the following Pohozaev identity holds for $u_{c} \in H^{1}\left(\mathbf{R}^{2}\right)$ weak solution of (1.4):

$$
\lambda \int_{\mathbf{R}^{2}}\left|u_{c}\right|^{2}-2 A\left(u_{c}\right)+2 \int_{\mathbf{R}^{2}} F\left(u_{c}\right)=0 .
$$

By multiplying (1.4) by $u_{c}$ and integrating, we derive a second identity

$$
\left\|\nabla u_{c}\right\|_{2}^{2}-\lambda \int_{\mathbf{R}^{2}}\left|u_{c}\right|^{2}+3 A\left(u_{c}\right)-\int_{\mathbf{R}^{2}} f\left(u_{c}\right) u_{c}=0 .
$$

Thus we have immediately

$$
\left\|\nabla u_{c}\right\|_{2}^{2}+A\left(u_{c}\right)-\int_{\mathbf{R}^{2}}\left(f\left(u_{c}\right) u_{c}-2 F\left(u_{c}\right)\right)=0,
$$

that is, $J\left(u_{c}\right)=0$. Then by Lemma $2.3,(2.27)$ and (2.28), we have

$$
\begin{aligned}
\lambda\left\|u_{c}\right\|^{2} & =\frac{2 p-6}{p-2} A\left(u_{c}\right)-\frac{2}{p-2}\left\|\nabla u_{c}\right\|_{2}^{2}+\frac{2}{p-2} \int_{\mathbf{R}^{2}}\left[f\left(u_{c}\right) u_{c}-p F\left(u_{c}\right)\right] \\
& \leq \frac{2 p-6}{p-2} A\left(u_{c}\right)-\frac{2}{p-2}\left\|\nabla u_{c}\right\|_{2}^{2} \\
& \leq\left(\frac{p-3}{8 \pi^{2}(p-2)}\left\|u_{c}\right\|^{2}-\frac{2}{p-2}\right)\left\|\nabla u_{c}\right\|_{2}^{2} \leq 0,
\end{aligned}
$$

if $\left\|u_{c}\right\|^{2}<\frac{4 \pi}{\sqrt{p-3}}$. Thus $u_{c}=0$. 
Lemma 2.12. For any $\lambda>0$ and $\kappa>0$, there exists no positive solution to (1.4) in $H_{r}^{1}\left(\mathbf{R}^{2}\right)$.

Proof. By (2.8), we have $f(\tau) \tau>0$ for $\tau \neq 0$. Then the rest of proof is similar to [16, Lemma 2.9] or [8, Proposition 4.2]. So we omit it.

Proof of Theorem 1.1. Point (i) follows form Lemma 2.6.

By Lemmas 2.5, 2.7 and 2.9, it is enough to show that for any $0<c \leq c_{*},\left.E\right|_{V(c)}$ attains its minimum at $u_{c}$, where $c_{*}$ is given by (2.21). Since $c_{*} \leq \frac{4 \pi}{\sqrt{2 p-4}}<\frac{4 \pi}{\sqrt{p-3}}$ for $p>4$, the first part of point (ii) follows from Lemmas 2.10-2.12. To end this, by $J\left(u_{c}\right)=0,(2.8),(2.10)$ and (2.11) yields

$$
\begin{aligned}
\left\|\nabla u_{c}\right\|_{2}^{2} & \leq \int_{\mathbf{R}^{2}}\left[f\left(u_{c}\right) u_{c}-2 F\left(u_{c}\right)\right] \leq \varepsilon\left\|u_{c}\right\|_{2}^{2}+C_{\varepsilon}\left\|u_{c}\right\|_{p}^{p} \\
& \leq \frac{\rho_{1}}{2}+C_{1}\left\|u_{c}\right\|_{p}^{p} \leq \frac{\rho}{2}+C_{2}\left\|\nabla u_{c}\right\|_{2}^{p-2} c
\end{aligned}
$$

where $\rho$ is given in Lemma 2.7. Thus,

$$
\left\|\nabla u_{c}\right\|_{2}^{2} \leq 2 C_{2}\left\|\nabla u_{c}\right\|_{2}^{p-2} c .
$$

Thanks to $p>4,(2.30)$ tells us that $\left\|\nabla u_{c}\right\|_{2} \rightarrow \infty$ as $c \rightarrow 0^{+}$. Moreover, we deduce from (2.29) that

$$
\lambda_{c} \leq \frac{1}{c}\left(\frac{p-3}{8 \pi^{2}(p-2)} c-\frac{2}{p-2}\right)\left\|\nabla u_{c}\right\|_{2}^{2} \leq-\frac{1}{c(p-2)}\left\|\nabla u_{c}\right\|_{2}^{2} \rightarrow-\infty,
$$

as $c \rightarrow 0^{+}$. Thus the proof is completed.

\section{Proof of the multiplicity results}

In this section, we shall prove Theorems 1.2-1.4. From now on, we assume that $\left(f_{1}\right)-\left(f_{5}\right)$ hold. Let $X=H_{r}^{1}\left(\mathbf{R}^{2}\right)$ and $\left\{V_{n}\right\} \subset X$ be a strictly increasing sequence of finite-dimensional linear subspaces such that $\bigcup_{n} V_{n}$ is dense in $X$. In addition, we denote the orthogonal space of $V_{n}$ in $X$ and the dual space of $X$ by $V_{n}^{\perp}$ and $X^{*}$, respectively.

Lemma 3.1. [1, Lemma 2.1] For $q>2$ there holds:

$$
\mu_{n}(q):=\inf _{u \in V_{n-1}^{\perp}} \frac{\int_{\mathbf{R}^{2}}\left(|\nabla u|^{2}+|u|^{2}\right)}{\left(\int_{\mathbf{R}^{2}}|u|^{q}\right)^{2 / q}}=\inf _{u \in V_{n-1}^{\perp}} \frac{\|u\|^{2}}{\|u\|_{q}^{2}} \rightarrow \infty, \quad \text { as } n \rightarrow \infty .
$$

Now for $c>0$ fixed and for each $n \in \mathbf{N}^{+}$, we define

$$
\varrho_{n}:=L^{-\frac{2}{p-2}} \mu_{n}(p)^{\frac{p}{p-2}} \quad \text { with } L=\max _{x>0} \frac{\left(x^{2}+c\right)^{p / 2}}{x^{p}+c^{p / 2}}
$$

and

$$
B_{n}:=\left\{u \in V_{n-1}^{\perp} \cap S_{r}(c):\|\nabla u\|_{2}^{2}=\varrho_{n}\right\} .
$$

Then we have:

Lemma 3.2. $b_{n}:=\inf _{u \in B_{n}} E_{\kappa}(u) \rightarrow \infty$ as $n \rightarrow \infty$. In particular, we can assume that $b_{n} \geq 1$ for any $n \in \mathbf{N}^{+}$without any restriction. 
Proof. For any $u \in B_{n}$, using (2.10), we get that

$$
\begin{aligned}
E_{\kappa}(u) & =\frac{1}{2}\|\nabla u\|_{2}^{2}+\frac{\kappa}{2} A\left(u_{n}\right)-\int_{\mathbf{R}^{2}} F(u) \geq \frac{1}{2}\|\nabla u\|_{2}^{2}-C_{1} c-\frac{1}{p}\|u\|_{p}^{p} \\
& \geq \frac{1}{2}\|\nabla u\|_{2}^{2}-C_{1} c-\frac{1}{p \mu_{n}(p)^{p / 2}}\left(\|\nabla u\|_{2}^{2}+c\right)^{\frac{p}{2}} \\
& \geq \frac{1}{2}\|\nabla u\|_{2}^{2}-C_{1} c-\frac{L}{p \mu_{n}(p)^{p / 2}}\left(\|\nabla u\|_{2}^{p}+c^{\frac{p}{2}}\right) \\
& =\left(\frac{1}{2}-\frac{1}{p}\right) \varrho_{n}-C_{1} c-\frac{L}{p \mu_{n}(p)^{p / 2}} c^{\frac{p}{2}}
\end{aligned}
$$

Combining this estimate and Lemma 3.1, we deduce from $p>4$ that $b_{n} \rightarrow \infty$ as $n \rightarrow \infty$.

Next we follow the arguments in [1] to set up a min-max scheme. Define a map $H: X \times \mathbf{R} \rightarrow X$ by $H(u, s)=e^{s} u\left(e^{s} x\right)$ and a $\mathcal{C}^{1}$ functional $\widetilde{E}_{\kappa}: X \times \mathbf{R} \rightarrow X$ given by

$$
\widetilde{E}_{\kappa}(u, s)=\frac{e^{2 s}}{2} \int_{\mathbf{R}^{2}}|\nabla u|^{2}+\frac{e^{2 s}}{2} A(u)-e^{-2 s} \int_{\mathbf{R}^{2}} F\left(e^{s} u\right) .
$$

It is clear that $\widetilde{E}_{\kappa}(u, s)=E_{\kappa}(H(u, s))$ and for all $s \in \mathbf{R}, H(u, s) \in S_{r}(c)$ if $u \in S_{r}(c)$. We know from Lemmas 2.3 and 3.1 that

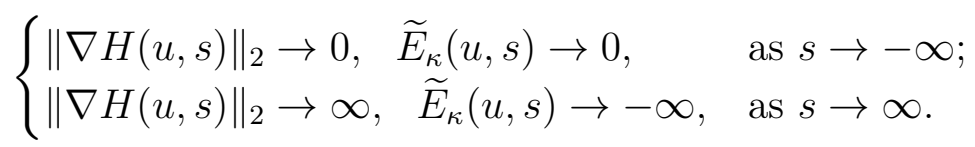

Thus by virtue of the fact $V_{n}$ is finite dimensional, for each $n \in \mathbf{N}^{+}$, there exists a $s_{n}>0$ such that

$$
\bar{g}_{n}:[0,1] \times\left(S_{r}(c) \cap V_{n}\right) \rightarrow S_{r}(c), \quad \bar{g}_{n}(t, u)=H\left(u,(2 t-1) s_{n}\right)
$$

satisfies

$$
\begin{cases}\left\|\nabla \bar{g}_{n}(0, u)\right\|_{2}<\varrho_{n}, & \left\|\nabla \bar{g}_{n}(1, u)\right\|_{2}>\varrho_{n} ; \\ E_{\kappa}\left(\bar{g}_{n}(0, u)\right)<b_{n}, & E_{\kappa}\left(\bar{g}_{n}(1, u)\right)<b_{n} .\end{cases}
$$

Now we define

$$
\begin{aligned}
\Gamma_{n}:=\{g: & {[0,1] \times\left(S_{r}(c) \cap V_{n}\right) \rightarrow S_{r}(c) \mid g \text { is continuous, odd in } u } \\
& \text { and such that } \left.\forall u: g(0, u)=\bar{g}_{n}(0, u), g(1, u)=\bar{g}_{n}(1, u)\right\} .
\end{aligned}
$$

Clearly $\bar{g}_{n} \in \Gamma_{n}$. Using the linking property (see [1, Lemma 2.3]), we have immediately the following intersection result:

Lemma 3.3. For each $n \in \mathbf{N}^{+}$,

$$
\gamma_{n}^{\kappa}(c):=\inf _{g \in \Gamma_{n}} \max _{t \in[0,1], u \in S_{r}(c) \cap V_{n}} E_{\kappa}(g(t, u)) \geq b_{n} .
$$

Next we will show that $\left\{\gamma_{n}^{\kappa}(c)\right\}$ is indeed a sequence of critical values of $E_{\kappa}$ on $S_{r}(c)$. To do that, we first show that there exists a bounded (PS) sequence at each level $\gamma_{n}^{\kappa}(c)$. We fix an arbitrary $n \in \mathbf{N}^{+}$from now on. To this end, we adopt the approach developed by [18], already applied in [1, 16]. Set

$$
\widetilde{\gamma}_{n}^{\kappa}(c)=\inf _{\tilde{g} \in \tilde{\Gamma}_{n}} \max _{t \in[0,1], u \in S_{r}(c) \cap V_{n}} \widetilde{E}_{\kappa}(\tilde{g}(t, u)),
$$


where

$$
\begin{gathered}
\widetilde{\Gamma}_{n}:=\left\{\tilde{g}:[0,1] \times\left(S_{r}(c) \cap V_{n}\right) \rightarrow S_{r}(c) \times \mathbf{R} \mid \tilde{g} \text { is continuous, odd in } u\right. \\
\text { and such that } \left.H \circ \tilde{g} \in \Gamma_{n}\right\} .
\end{gathered}
$$

Clearly, for any $g \in \Gamma_{n}, \tilde{g}:=(g, 0) \in \widetilde{\Gamma}_{n}$. From the fact that the maps

$$
\varphi: \Gamma_{n} \rightarrow \widetilde{\Gamma}_{n}, \quad g \mapsto \varphi(g):=(g, 0) \text { and } \psi: \widetilde{\Gamma}_{n} \rightarrow \Gamma_{n}, \quad \tilde{g} \mapsto \psi(\tilde{g}):=H \circ \tilde{g}
$$

satisfy

$$
\widetilde{E}_{\kappa}(\varphi(g))=E_{\kappa}(g) \quad \text { and } \quad E_{\kappa}(\psi(\tilde{g}))=\widetilde{E}_{\kappa}(\tilde{g})
$$

we get that $\tilde{\gamma}_{n}^{\kappa}(c)=\gamma_{n}^{\kappa}(c)$. Following [3] or [31, page 86], the tangent space at a point $(u, s) \in S_{r}(c) \times \mathbf{R}$ is defined as $\widetilde{T}_{(u, s)}=\left\{(v, t) \in Y: \int_{\mathbf{R}^{2}} u v=0\right\}$, where $Y:=X \times \mathbf{R}$ is equipped with the scalar product $((u, s),(v, t))_{Y}=\int_{\mathbf{R}^{2}}(\nabla u \nabla v+u v+s t)$ and the corresponding norm defined by $\|(u, s)\|_{Y}=\left(\|u\|^{2}+s^{2}\right)^{1 / 2}$. Then the norm of the derivative of $\left.\widetilde{E}_{\kappa}\right|_{S_{r}(c) \times \mathbf{R}}$ at $(u, s)$ is defined by

$$
\left\|\left(\left.\widetilde{E}_{\kappa}\right|_{S_{r}(c) \times \mathbf{R}}\right)^{\prime}(u, s)\right\|_{*}=\sup _{(u, s) \in \widetilde{T}_{(u, s)},\|(u, s)\|_{Y}=1}\left|\left\langle E_{\kappa}^{\prime}(u, s),(v, t)\right\rangle\right| .
$$

Lemma 3.4. For any fixed $c>0$ and $n \in \mathbf{N}^{+}$, there exists a sequence $\left\{v_{d}\right\} \subset$ $S_{r}(c)$ such that as $d \rightarrow \infty$,

$$
\left\{\begin{array}{l}
E_{\kappa}\left(v_{d}\right) \rightarrow \gamma_{n}^{\kappa}(c), \\
\left\|\left.E_{\kappa}^{\prime}\right|_{S_{r}(c)}\left(v_{d}\right)\right\|_{*} \rightarrow 0 \\
J_{\kappa}\left(v_{d}\right) \rightarrow 0
\end{array}\right.
$$

Proof. We borrow some elements of Lemma 2.4 in [18]. Noting the definition of $\gamma_{n}^{\kappa}(c)$, we have that for each $d \in \mathbf{N}^{+}$, there exists $g_{d} \in \Gamma_{n}$ such that

$$
\max _{t \in[0,1], u \in S_{r}(c) \cap V_{n}} E_{\kappa}\left(g_{d}(t, u)\right) \leq \gamma_{n}^{\kappa}(c)+\frac{1}{d} .
$$

Set $\tilde{g}_{d}=\left(g_{d}, 0\right)$, then $\tilde{g}_{d} \in \tilde{\Gamma}_{n}$ and

$$
\max _{t \in[0,1], u \in S_{r}(c) \cap V_{n}} \widetilde{E}_{\kappa}\left(\tilde{g}_{d}(t, u)\right) \leq \tilde{\gamma}_{n}^{\kappa}(c)+\frac{1}{d}
$$

since $\tilde{\gamma}_{n}^{\kappa}(c)=\gamma_{n}^{\kappa}(c)$. Similar to Proposition 2.2 in [18], we obtain a sequence $\left\{\left(u_{d}, s_{d}\right)\right\} \subset S_{r}(c) \times \mathbf{R}$ satisfying that

$$
\widetilde{E}_{\kappa}\left(u_{d}, s_{d}\right) \rightarrow \gamma_{n}^{\kappa}(c), \quad\left\|\left(\left.\widetilde{E}_{\kappa}\right|_{S_{r}(c) \times \mathbf{R}}\right)^{\prime}\left(u_{d}, s_{d}\right)\right\|_{*} \rightarrow 0
$$

and

$$
\min _{t \in[0,1], u \in S_{r}(c) \cap V_{n}}\left\|\left(u_{d}, s_{d}\right)-\tilde{g}_{d}(t, u)\right\|_{Y} \rightarrow 0 .
$$

For each $d \in \mathbf{N}^{+}$, let $v_{d}=H\left(u_{d}, s_{d}\right)$. Then $v_{d} \in S_{r}(c)$ and from (3.9), we have that

$$
E_{\kappa}\left(v_{d}\right) \rightarrow \gamma_{n}^{\kappa}(c) \quad \text { as } d \rightarrow \infty .
$$


For any $(w, t) \in Y$, we see that

$$
\begin{aligned}
\left\langle\widetilde{E}_{\kappa}^{\prime}\left(u_{d}, s_{d}\right),(w, t)\right\rangle & \\
= & t e^{2 s_{d}}\left\|\nabla u_{d}\right\|_{2}^{2}+e^{2 s_{d}} \int_{\mathbf{R}^{2}} \nabla u_{d} \nabla w+\kappa e^{2 s_{d}} \int_{\mathbf{R}^{2}} \frac{u_{d} w}{|x|^{2}}\left(\int_{0}^{|x|} \frac{r}{2} u_{d}^{2}(r) d r\right)^{2} \\
& +\kappa e^{2 s_{d}} \int_{\mathbf{R}^{2}} \frac{\left|u_{d}\right|^{2}}{|x|^{2}}\left(\int_{0}^{|x|} \frac{r}{2} u_{d}^{2}(r) d r\right)\left(\int_{0}^{|x|} r u_{d}(r) w(r) d r\right)+t \kappa e^{2 s_{d}} A\left(u_{d}\right) \\
& -t e^{-2 s_{d}} \int_{\mathbf{R}^{2}}\left[f\left(e^{s_{d}} u_{d}\right) e^{s_{d}} u_{d}-2 F\left(e^{s_{d}} u_{d}\right)\right]-e^{-2 s_{d}} \int_{\mathbf{R}^{2}} f\left(e^{s_{d}} u_{d}\right) e^{s_{d}} w \\
= & \left\langle E_{b}^{\prime}\left(v_{d}\right), H\left(w, s_{d}\right)\right\rangle+J_{\kappa}\left(v_{d}\right) t .
\end{aligned}
$$

Let $(w, t)=(0,1) \in \widetilde{T}_{\left(u_{d}, s_{d}\right)}$, then (3.9) and (3.12) imply that

$$
J_{\kappa}\left(v_{d}\right) \rightarrow 0 .
$$

For any $w \in T_{v_{d}}$, if we take $t=0$ in (3.12), then we get that

$$
\left\langle\widetilde{E}_{\kappa}^{\prime}\left(u_{d}, s_{d}\right),\left(H\left(w,-s_{d}\right), 0\right)\right\rangle=\left\langle E_{\kappa}^{\prime}\left(v_{d}\right), w\right\rangle,
$$

where $T_{v_{d}}=\left\{w \in X: \int_{\mathbf{R}^{2}} w v_{d}=0\right\}$. Moreover, since $\int_{\mathbf{R}^{2}} w v_{d}=\int_{\mathbf{R}^{2}} u_{d} H\left(w,-s_{d}\right)$, we obtain that $w \in T_{v_{d}} \Leftrightarrow\left(H\left(w,-s_{d}\right), 0\right) \in \widetilde{T}_{\left(u_{d}, s_{d}\right)}$. To verify that $\left\|\left.E_{\kappa}^{\prime}\right|_{S_{r}(c)}\left(v_{d}\right)\right\|_{*} \rightarrow 0$, it suffices to show that $\left\{\left(H\left(w,-s_{d}\right), 0\right)\right\}$ is uniformly bounded in $Y$ for $n$ large, which is insured by the fact that

$$
\left|s_{d}\right|=\left|s_{d}-0\right| \leq \min _{t \in[0,1], u \in S_{r}(c) \cap V_{n}}\left\|\left(u_{d}, s_{d}\right)-\left(g_{d}(t, u), 0\right)\right\|_{Y} \leq 1
$$

for $d$ large enough. This ends the proof.

Proposition 3.5. Let $c \in\left(0, \frac{4 \pi}{\sqrt{p-3}}\right), \kappa \in(0, p-3)$ and $\left\{u_{d}^{n}\right\} \subset S_{r}(c)$ be a sequence satisfying (3.8). Then $\left\{u_{d}^{n}\right\} \subset S_{r}(c)$ is bounded. Moreover, there exist $\left\{\lambda_{d}^{n}\right\} \subset \mathbf{R}$ and $u_{n} \in X$, such that, up to subsequence, as $d \rightarrow \infty$,

(i) $u_{d}^{n} \rightarrow u_{n} \neq 0$ in $X$;

(ii) $\lambda_{d}^{n} \rightarrow \lambda_{n}$ in $\mathbf{R}$;

(iii) $E_{\kappa}^{\prime}\left(u_{d}^{n}\right)-\lambda_{d}^{n} u_{d}^{n} \rightarrow 0$ in $X^{*}$.

(iv) $E_{\kappa}^{\prime}\left(u_{n}\right)-\lambda_{n} u_{n}=0$ in $X^{*}$;

In addition, if $\lambda_{n}<0$, then we have $u_{d} \rightarrow u_{n}$ in $X$ as $d \rightarrow \infty$. In particular, $\left\|u_{n}\right\|_{2}^{2}=c$ and $E_{\kappa}\left(u_{n}\right)=\gamma_{n}^{\kappa}(c)$.

Proof. By the second relation of (3.8), we obtain

$$
\left\langle E_{\kappa}^{\prime}\left(u_{d}^{n}\right), u_{d}^{n}\right\rangle=\left\|\nabla u_{d}^{n}\right\|_{2}^{2}+3 \kappa A\left(u_{d}^{n}\right)-\int_{\mathbf{R}^{2}} f\left(u_{d}^{n}\right) u_{d}^{n}=o\left(\left\|u_{d}^{n}\right\|\right) .
$$

From Lemma 2.3, (2.7), (3.8) and (3.15), we conclude

$$
\begin{aligned}
\gamma_{n}^{\kappa}(c)+o(1) & =\frac{1}{2}\left\|\nabla u_{d}^{n}\right\|_{2}^{2}+\frac{\kappa}{2} A\left(u_{d}^{n}\right)-\int_{\mathbf{R}^{2}} F\left(u_{d}^{n}\right) \\
& \geq \frac{1}{2}\left\|\nabla u_{d}^{n}\right\|_{2}^{2}+\frac{\kappa}{2} A\left(u_{d}^{n}\right)-\frac{1}{4}\left(\left\|\nabla u_{d}^{n}\right\|+3 \kappa A\left(u_{d}^{n}\right)\right)+o\left(\left\|u_{d}^{n}\right\|\right) \\
& =\frac{1}{4}\left(\left\|\nabla u_{d}^{n}\right\|_{2}^{2}-\kappa A\left(u_{d}^{n}\right)\right)+o\left(\left\|u_{d}^{n}\right\|\right) \\
& \geq \frac{1}{4}\left(1-\frac{\kappa c^{2}}{16 \pi^{2}}\right)\left\|\nabla u_{d}^{n}\right\|_{2}^{2}+o\left(\left\|u_{d}^{n}\right\|\right) .
\end{aligned}
$$


Thanks to $c \in\left(0, \frac{4 \pi}{\sqrt{p-3}}\right)$ and $\kappa \in(0, p-3),(3.16)$ shows that $\left\{u_{d}^{n}\right\} \subset S_{r}(c)$ is bounded. Similar to the proof of Lemma 2.9, we know that there exists $u_{n} \neq 0$ such that, taking a subsequence if necessary, as $d \rightarrow \infty$,

$$
\begin{cases}u_{d}^{n} \rightarrow u_{n} & \text { in } X \\ u_{d}^{n} \rightarrow u_{n} & \text { in } L^{q}\left(\mathbf{R}^{2}\right), \\ u_{d}^{n} \rightarrow u_{n} & \text { a.e. in } \mathbf{R}^{2},\end{cases}
$$

for $q \in(2, \infty)$. Thus point (i) holds and

$$
\int_{\mathbf{R}^{2}} f\left(u_{d}^{n}\right) u_{d}^{n} \rightarrow f\left(u_{n}\right) u_{n} \text { and } \int_{\mathbf{R}^{2}} F\left(u_{d}^{n}\right) \rightarrow F\left(u_{n}\right) .
$$

In addition, the proofs of points (ii)-(iv) are similar to that in [16, Proposition 2.26]. Using (ii)-(iv), we obtain that

$$
\left\langle E_{\kappa}^{\prime}\left(u_{d}^{n}\right)-\lambda_{d}^{n} u_{d}^{n}, u_{d}^{n}-u_{n}\right\rangle=o(1) \quad \text { and } \quad\left\langle E_{\kappa}^{\prime}\left(u_{n}\right)-\lambda_{n} u_{n}, u_{d}^{n}-u_{n}\right\rangle=o(1) .
$$

if $\lambda_{n}<0$, then we deduce from (3.18), (3.19) and Lemma 2.2 that

$$
\left\|\nabla u_{d}\right\|_{2} \rightarrow\left\|\nabla u_{n}\right\|_{2} \quad \text { and } \quad\left\|u_{d}\right\|_{2} \rightarrow\left\|u_{n}\right\|_{2}, \quad \text { as } d \rightarrow \infty \text {. }
$$

The proof is completed.

Remark 3.1. In the above proof, (3.16) also shows that if $\kappa>0$ is sufficient small, then $\left\{u_{d}^{n}\right\} \subset S_{r}(c)$ is bounded and thus the the conclusions (i)-(iv) of Proposition 3.5 still hold.

Proof of Theorem 1.2. From Lemmas 2.11 and 3.4 and Proposition 3.5, it follows that for $c \in\left(0, \frac{4 \pi}{\sqrt{p-3}}\right), \kappa \in(0, p-3)$ and each $n \in \mathbf{N}^{+}$, there exists a couple of solutions $\left(u_{n}, \lambda_{n}\right) \in S_{r}(c) \times \mathbf{R}^{-}$for (1.4) with $E_{\kappa}\left(u_{n}\right)=\gamma_{n}^{\kappa}(c)$. By Lemmas 3.2 and 3.3 , we deduce that $\gamma_{n}(c) \rightarrow \infty$ as $n \rightarrow \infty$ and the sequences of solutions $\left\{\left(u_{n}, \lambda_{n}\right)\right\}$ is unbounded. Thus (i) is proved.

To prove (ii), we only need to show that if $\left(u_{n}, \lambda_{n}\right) \in H_{r}^{1}\left(\mathbf{R}^{2}\right) \times \mathbf{R}$ solves (1.4), then $\lambda_{n}<0$ provided that $\kappa>0$ is sufficient small. In fact, similar to (2.29), we get that

$$
\lambda_{n} c \leq \frac{p-3}{8 \pi^{2}(p-2)}\left(\kappa c-\frac{16 \pi^{2}}{p-3}\right)\left\|\nabla u_{c}\right\|_{2}^{2}<0,
$$

for $\kappa>0$ small enough. This ends the proof.

Remark 3.2. In the proof of Theorem 1.2 (ii), $\kappa=0$ is allowed. Therefore, we can prove Corollary 1.3.

Proof of Theorem 1.4. For $\kappa>0$ sufficiently small, let $\left\{\left(u_{n}^{\kappa}, \lambda_{n}^{\kappa}\right)\right\} \subset S_{r}(c) \times \mathbf{R}^{-}$ be obtained in Theorem 1.3 (ii). We decare that for any sequence $\left\{\kappa_{m}\right\} \rightarrow 0^{+},\left\{u_{n}^{\kappa_{m}}\right\}$ is bounded in $X$. Indeed, it follows from $\operatorname{dim} V_{n}<\infty$ that for each $n \in \mathbf{N}^{+}$,

$$
\gamma_{n}^{\kappa}(c):=\inf _{g \in \Gamma_{n}} \max _{t \in[0,1], u \in S_{r}(c) \cap V_{n}} E_{\kappa}(g(t, u)) \leq \inf _{g \in \Gamma_{n}} \max _{t \in[0,1], u \in S_{r}(c) \cap V_{n}} E_{1}(g(t, u))<\infty .
$$

Since the sequence of $\left\{\left(u_{n}^{\kappa_{m}}, \lambda_{n}^{\kappa_{m}}\right)\right\}_{m \in \mathbf{N}} \subset S_{r}(c) \times \mathbf{R}^{-}$solves (1.4), we get that $\left\{u_{n}^{\kappa_{m}}\right\} \subset$ $V(c)$ and

$$
\lambda_{n}^{\kappa_{m}}=\frac{1}{c}\left[\left\|\nabla u_{n}^{\kappa_{m}}\right\|_{2}^{2}+3 \kappa_{m} A\left(u_{n}^{\kappa_{m}}\right)-\int_{\mathbf{R}^{2}} f\left(u_{n}^{\kappa_{m}}\right) u_{n}^{\kappa_{m}}\right] .
$$

Similar to Remark 3.1, we can obtain $\left\{u_{n}^{\kappa_{m}}\right\}$ is bounded in $X$. Furthermore, by (2.9)-(2.11) and Lemmas 2.1 and 2.3, it is easy to see that $\left\{\lambda_{n}^{\kappa_{m}}\right\}$ is bounded in $\mathbf{R}$. 
Then there exist a subsequence of $\left\{\kappa_{m}\right\}$, still denoted by $\left\{\kappa_{m}\right\}, u_{n}^{0} \in X$ and $\lambda_{n}^{0} \leq 0$ such that as $m \rightarrow \infty, \lambda_{n}^{\kappa_{m}} \rightarrow \lambda_{n}^{0}$ and

$$
\begin{cases}u_{n}^{\kappa_{m}} \rightarrow u_{n}^{0} & \text { in } X ; \\ u_{n}^{\kappa_{m}} \rightarrow u_{n}^{0} & \text { in } L^{q}\left(\mathbf{R}^{2}\right), 2<q<6 ; \\ u_{n}^{\kappa_{m}} \rightarrow u_{n}^{0} & \text { a.e. in } \mathbf{R}^{2} .\end{cases}
$$

It is trivial that for each $n \in \mathbf{N}^{+},\left(u_{n}^{0}, \lambda_{n}^{0}\right)$ is a couple of solutions of (1.13), i.e.,

$$
\int_{\mathbf{R}^{2}} \nabla u_{n}^{0} \nabla v-\lambda_{n}^{0} \int_{\mathbf{R}^{2}} u_{n}^{0} v=\int_{\mathbf{R}^{2}} f\left(u_{n}^{0}\right) v, \quad \forall v \in X
$$

Then we have

$$
\int_{\mathbf{R}^{2}} \nabla u_{n}^{0} \nabla\left(u_{n}^{\kappa_{m}}-u_{n}^{0}\right)-\lambda_{n}^{0} \int_{\mathbf{R}^{2}} u_{n}^{0}\left(u_{n}^{\kappa_{m}}-u_{n}^{0}\right)=\int_{\mathbf{R}^{2}} f\left(u_{n}^{0}\right)\left(u_{n}^{\kappa_{m}}-u_{n}^{0}\right) .
$$

Since $\left\{\left(u_{n}^{\kappa}, \lambda_{n}^{\kappa}\right)\right\}$ is a sequence of couples of solutions for (1.4) with $\kappa=\kappa_{m}$ and $\left\{u_{n}^{\kappa_{m}}\right\}$ is bounded in $X$, using $\lambda_{n}^{\kappa_{m}} \underset{m}{\rightarrow} \lambda_{n}^{0}$ and Lemma 2.3, we get that

$$
\int_{\mathbf{R}^{2}} \nabla u_{n}^{\kappa_{m}} \nabla\left(u_{n}^{\kappa_{m}}-u_{n}^{0}\right)-\lambda_{n}^{0} \int_{\mathbf{R}^{2}} u_{n}^{\kappa_{m}}\left(u_{n}^{\kappa_{m}}-u_{n}^{0}\right)=\int_{\mathbf{R}^{2}} f\left(u_{n}^{\kappa_{m}}\right)\left(u_{n}^{\kappa_{m}}-u_{n}^{0}\right)+o(1) .
$$

(3.21)-(3.23) imply that

$$
\left\|\nabla\left(u_{n}^{\kappa_{m}}-u_{n}^{0}\right)\right\|_{2}^{2}-\lambda_{n}^{0}\left\|u_{n}^{\kappa_{m}}-u_{n}^{0}\right\|_{2}^{2}=o(1) .
$$

At this point, using $\lambda_{n}^{0} \leq 0$ we get $\left\|\nabla\left(u_{n}^{\kappa_{m}}-u_{n}^{0}\right)\right\|_{2} \rightarrow 0$. Moreover, if $\lambda_{n}^{0}=0$, then $\left(u_{n}^{0}, 0\right)$ is a couple of solutions of (1.13). Thus it is readily checked that $\int_{\mathbf{R}^{2}} F\left(u_{n}^{0}\right)=0$ and $\int_{\mathbf{R}^{2}} f\left(u_{n}^{0}\right) u_{n}^{0}=\left\|\nabla u_{n}^{0}\right\|_{2}^{2}=0$. Then by Lemma 3.3, as $m \rightarrow \infty$,

$$
1 \leq b_{n} \leq \gamma_{n}^{\kappa_{m}}(c)=E_{\kappa_{m}}\left(u_{n}^{\kappa_{m}}\right) \rightarrow 0 \text {. }
$$

This contradiction means $\lambda_{n}^{0}<0$. From (3.24), we deduce that $\left\|u_{n}^{\kappa_{m}}-u_{n}^{0}\right\|_{2} \rightarrow 0$. Hence the sequence of $\left\{\left(u_{n}^{0}, \lambda_{n}^{0}\right)\right\} \subset S_{r}(c) \times \mathbf{R}^{-}$solves (1.13).

Acknowledgements. The authors would like to thank the referees for their valuable comments and suggestions. H. B. Chen is supported by the National Natural Science Foundation of China (No. 11671403).

\section{References}

[1] Bartsch, T., and S. De Valeriola: Normalized solutions of nonlinear Schrödinger equations. - Arch. Math. 100, 2013, 75-83.

[2] Bartsch, T., L. Jeanjean, and N. Soave: Normalized solutions for a system of coupled cubic Schrödinger equations on $\mathbf{R}^{3}$. - J. Math. Pures Appl. 106:4, 2016, 583-614.

[3] Bellazzini, J., L. Jeanjean, and T. J. Luo: Existence and instability of standing waves with prescribed norm for a class of Schrödinger-Poisson equations. - Proc. London Math. Soc. $107: 3,2013,303-339$.

[4] Bellazzini, J., and G. Siciliano: Scaling properties of functionals and existence of constrained minimizers. - J. Funct. Anal. 261:9, 2011, 2486-2507.

[5] Bellazzini, J., and G. Siciliano: Stable standing waves for a class of nonlinear SchrödingerPoisson equations. - Z. Angew. Math. Phys. 62:2, 2011, 267-280.

[6] Berestycki, H., and P. L. Lions: Nonlinear scalar field equations I. - Arch. Ration. Mech. Anal. 82, 1983, 313-345.

[7] CAO, D., and X. ZHU: On the existence and nodal character of semilinear elliptic equations. - Acta Math. Sci. 8, 1988, 345-359. 
[8] Byeon, J., H. Huh, and J. Seok: Standing waves of nonlinear Schrödinger equations with the gauge field. - J. Funct. Anal. 263, 2012, 1575-1608.

[9] Byeon, J., H. Huh, and J. Seok: On standing waves with a vortex point of order $N$ for the nonlinear Chern-Simons-Schrödinger equations. - J. Differential Equations 261, 2016, 12851316.

[10] Deng, Y., S. Peng, and W. Shuai: Nodal standing waves for a gauged nonlinear Schrödinger equation in $\mathbf{R}^{2}$. - J. Differential Equations 264, 2018, 4006-4035.

[11] HuH, H.: Blow-up solutions of the Chern-Simons-Schrödinger equations. - Nonlinearity 22, 2009, 967-974.

[12] HuH, H.: Standing waves of the Schrödinger equation coupled with the Chern-Simons gauge field. - J. Math. Phys. 53, 2012, 063702.

[13] JACKIW, R., and S. Y. PI: Soliton solutions to the gauged nonlinear Schrödinger equations. Phys. Rev. Lett. 64, 1990, 2969-2972.

[14] JACKIw, R., and S. Y. PI: Classical and quantal nonrelativistic Chern-Simons theory. - Phys. Rev. D 42, 1990, 3500-3513.

[15] Jiang, Y., A. Pomponio, and D. Ruiz: Standing waves for a gauged nonlinear Schrödinger equation with a vortex point. - Commun. Contemp. Math. 18, 2016, 1-20.

[16] LI, G., and X. LuO: Normalized solutions for the Chern-Simons-Schrödinger equation in $\mathbf{R}^{2}$. - Ann. Acad. Sci. Fenn. Math. 42, 2017, 405-428.

[17] Li, G., X. Luo, and W. ShuAI: Sign-changing solutions to a gauged nonlinear Schrödinger equation. - J. Math. Anal. Appl. 455, 2017, 1559-1578.

[18] JEAnJEAN, L.: Existence of solutions with prescribed norm for semilinear elliptic equations. Nonlinear Anal. 28:10, 1997, 1633-1659.

[19] Jeanjean, L., and T. J. LuO: Sharp nonexistence results of prescribed $L^{2}$-norm solutions for some class of Schrödinger-Poisson and quasi-linear equations. - Z. Angrew. Math. Phys. 64, 2013, 937-954.

[20] LuO, T. J.: Multiplicity of normalized solutions for a class of nonlinear Schrödinger-PoissonSlater equations. - J. Math. Anal. Appl. 416, 2014, 195-204.

[21] LuO, X., and Q.F. WANG: Existence and asymptotic behavior of high energy normalized solutions for the Kirchhoff type equations in $\mathbf{R}^{3}$. - Nonlinear Anal. Real World Appl. 33, 2017, 19-32.

[22] Pomponio, A., and D. Ruiz: A variational analysis of a gauged nonlinear Schrödinger equation. - J. Eur. Math. Soc. 17, 2015, 1463-1486.

[23] Pomponio, A., and D. Ruiz: Boundary concentration of a Gauged nonlinear Schrödinger equation on large balls. - Calc. Var. Partial Differential Equations 53, 2015, 289-316.

[24] RuIz, D.: The Schrödinger-Poisson equation under the effect of a nonlinear local term. - J. Funct. Anal. 237, 2006, 655-674.

[25] SEOK, J.: Infinitely many standing waves for the nonlinear Chern-Simons-Schrödinger equations. - Advances in Math. Phys., 2015.

[26] Struwe, M.: Variational methods. Applications to nonlinear partial differential equations and Hamiltonian systems. - Ergeb. Math. Grenzgeb. (3) 34, third edition, Springer-Verlag, Berlin, 1996.

[27] TANG, X., and S. Chen: Ground state solutions of Nehari-Pohozaev type for SchrödingerPoisson type problems with general potentials. - Discrete Contin. Dyn. Syst. 37:9, 2017, 49735002.

[28] TANG, X., J. ZhANG, and W. ZhANG: Existence and concentration of solutions for the ChernSimons-Schrödinger system with general nonlinearity. - Results Math. 71, 2017, 643-655.

[29] Wan, Y.Y., and J.G. TAN: Standing waves for the Chern-Simons-Schrödinger systems without (AR) condition. - J. Math. Anal. Appl. 415, 2014, 422-434. 
[30] Weinstein, M. I.: Nonlinear Schrödinger equations and sharp interpolations estimates. - Commun. Math. Phys. 87, 1983, 567-576.

[31] Willem, M.: Minimax theorems. - Birkhäuser, Berlin, 1996.

[32] Xie, W.H., and C. Chen: Sign-changing solutions for the nonlinear Chern-SimonsSchrödinger equations. - Appl. Anal. DOI:10.1080/00036811.2018.1514020.

[33] YuAN, J.: Multiple normalized solutions of Chern-Simons-Schrödinger system. - Nonlinear Differ. Equ. Appl. 22, 2015, 1801-1816.

[34] YE, H. Y.: The sharp existence of constrained minimizers for a class of nonlinear Kirchhoff equations. - Math. Methods Appl. Sci. 38, 2014, 2663-2679.

Received 9 April 2018• Revised received 17 May $2019 \bullet$ Accepted 3 July 2019 